\title{
ЗНАЧЕНИЕ КРАЯ РЕЗЕКЦИИ В МУЛЬТИДИСЦИПЛИНАРНОМ ПОДХОДЕ ПРИ ЛЕЧЕНИИ РАКА МОЛОЧНОЙ ЖЕЛЕЗЫ
}

\author{
Куланбаев Е.М.․, Макимбетов Э.К. ${ }^{1}$ \\ ${ }^{1}$ Кыргызско-Российский Славянский университет, Бишкек, e-mail: makimbetov@rambler.ru
}

\begin{abstract}
Рак молочной железы (РМЖ) в настоящее время занимает лидирующее место среди заболеваний женского населения планеты, особенно в возрастной группе старше 50 лет. Так, из 10 млн выявляемых в мире онкологических заболеваний РМЖ составляет $>10 \%$, а за последнее десятилетие данная патология превзошла по частоте встречаемости рак шейки матки. Особенно четко эта закономерность прослеживается в экономически развитых странах, где заболеваемость достигает 27\%. Консервативная хирургия по сохранению груди с последующим облучением всей груди является стандартом лечения РМЖ на ранней стадии на протяжении уже более 25 лет. Со временем частота местных рецидивов (в сроки до 10 лет) неуклонно снижалась и в настоящее время составляет < 10\% для женщин с инвазивным раком. Проведен литературный обзор касательно края резекции при РМЖ по источникам Рubmed с поиском ключевых слов с 2000 по 2015 гг. До сих пор нет единого мнения, каким должен быть размер края резекции опухоли. С учетом высокой частоты повторных иссечений после первой операции анализированы рандомизированные, случай-контрольные и когортные исследования зарубежных авторов. Показано, что ширина края резекции величиной 0,8 см является недостаточной и не исключает необходимости повторного иссечения при инвазивной карциноме. В $41 \%$ случаев остаточная карцинома присутствовала на расстоянии $>2$ см. Наличие этой остаточной опухоли дает основание для использования лучевой терапии после сохранной операции.
\end{abstract}

Ключевые слова: рак молочной железы, край резекции, органосохранное лечение, местный рецидив.

\section{THE IMPORTANCE OF THE RESECTION MARGIN AT THE MULTIDISCIPLINARY APPROACH IN THE TREATMENT OF BREAST CANCER}

\author{
Kulanbayev M.E. ${ }^{1}$, Makimbetov E.K. ${ }^{1}$ \\ ${ }^{1}$ Kyrgyz-Russian Slavic University, Bishkek, e-mail: makimbetov@rambler.ru
}

Breast cancer (BC) is currently the leading disease among the female population of the planet, especially in the age group over 50 years. For example, out of 10 million cancer detected in the world, $\mathrm{BC}$ is $>10 \%$, and over the past decade, this pathology has surpassed the incidence of cervical cancer. This pattern is especially clear in economically developed countries, where the incidence reaches $27 \%$. Conservative breast preservation surgery followed by radiation of the entire breast is a standard treatment option for $\mathrm{BC}$ at an early stage for more than 25 years. Over time, the incidence of local relapses (up to 10 years) has been steadily declining and is now $<10 \%$ for women with invasive cancer. There is performed the literature review regarding margin of resection for $\mathrm{BC}$ by the sources of Pubmed with search keywords during 2000-2015. There is still no consensus on what should be the size of the resection margin of a tumor, high frequency of re-excision after initial surgery, authors analyzed randomized, case-control and cohort studies of foreign sources. It is shown that the resection edge width of 0.8 $\mathrm{cm}$ is insufficient and does not exclude the need for re-excision in invasive carcinoma. In $41 \%$ of cases, residual carcinoma was present at a distance of $>\mathbf{2 m}$ and the presence of this residual tumor provides the rationale for the use of RT after surgery.

Keywords: breast cancer, resection edge, organ-preserved treatment, local relapse.

Рак молочной железы (РМЖ) в настоящее время занимает лидирующее место среди заболеваний женского населения планеты, особенно в возрастной группе старше 50 лет. Так, из 10 млн выявляемых в мире онкологических заболеваний РМЖ составляет $>10 \%$, а за последнее десятилетие данная патология превзошла по частоте встречаемости рак шейки матки. Консервативная хирургия по сохранению груди с последующим облучением всей груди является стандартным вариантом лечения рака молочной железы (РМЖ) на ранней 
10 лет) неуклонно снижалась и в настоящее время составляет $<10 \%$ для женщин с инвазивным раком [1, с. 2466, 2, с. 2028] и несколько выше - для протокового рака [3, с. 624]. Фундаментальной частью сохранной операции является удаление опухоли с краем окружающей нормальной ткани молочной железы. На протяжении нескольких лет размер ширины края резекции остается предметом спора для многих клиницистов. В проспективных триалах установлена безопасность грудьсохраняющей хирургии и лучевой терапии (ЛТ) для инвазионного рака без использования маркировки опухоли. Другие исследования показывали только рост опухоли, поэтому фактическая микроскопическая ширина края резекции была неизвестна.

Цель исследования - изучить значение края резекции при мультидисциплинарном подходе в лечении рака молочной железы по данным литературы.

Использованы поисковые системы Google Scolar, The Lancet.com, Pubmed и иные с 2000 по 2015 гг. с использованием ключевых слов: «рак молочной железы», «край резекции», «органосохранное лечение», «местный рецидив». При этом были систематизированы несколько клинических триалов, проспективных и когортных метаанализов.

Края резекции - это участки ткани молочной железы, находящиеся на границе с операционным разрезом. При морфологическом исследовании края резекции даются следующие характеристики: позитивные края - раковые клетки обнаруживаются в краях иссеченных тканей; негативные края - раковые клетки не обнаруживаются в краях иссеченных тканей (в пределах 1 мм от края опухоли); «тесные» («закрытые») края промежуточная ситуация (опухоль в пределах 1 мм и менее от края операционного разреза). Морфолог при изучении препарата должен указать макроскопическое и микроскопическое расположение опухоли от края. Препараты готовят таким образом, чтобы визуализировалось расстояние от края опухоли до границы хирургического препарата, т. е. ширина резекции, которая определяет риск рецидива.

С течением времени, по мере того как хирурги и онкологи-радиологи искали способы для уменьшения частоты местного рецидива после сохранной операции, произошли определенные изменения в отношении края резекции. Был проведен популяционный опрос 318 хирургов. 50\% из них считают, что ширина края резекции величиной 0,8 см является недостаточной и не исключает необходимости повторного иссечения при инвазивной карциноме [4, с. 563]. И поэтому из-за отсутствия консенсуса стало обычной процедурой повторное иссечение после первоначальной лампэктомии. По результатам анализа популяционной выборки из 1468 пациентов, получивших сохранную хирургию в 2005-2007 гг., Morrow et al. сообщили, что 38\% из них перенесли дополнительную операцию после первой лампэктомии; повторное иссечение понадобилось в $26 \%$ случаев и мастэктомия - в 
12\% [5, с. 1 556]. В другой выборке (n=2 206) 23\% пациентов имели реэксцизию после лампэктомии [6, с. 475], причем у половины был отрицательный край резекции.

Стандартной методики оценки края резекции не существует, как и стандартного количества гистологических срезов, которые исследуются с каждой поверхности краев. Поля могут быть оценены с помощью радиального (перпендикулярного) метода или метода бритья (en face). Метод оценки краев резекции, как известно, не коррелирует с частотой местного контроля [7, с. 505].

При рассмотрении вопроса о том, что представляет собой соответствующий запас, полезно помнить, что отрицательный запас независимо от того, как он определен, не указывает на отсутствие остаточной карциномы в груди. Holland et al. использовали последовательное поперечное сечение для исследования 264 образцов тканей опухоли после мастэктомии, содержащих клинически и маммографически одноцентрические опухоли размером 4 см или менее. Только в $39 \%$ случаев микроскопическая опухоль ограничивалась клинически выраженной опухолью, а в $41 \%$ случаев остаточная карцинома присутствовала на расстоянии $>2$ см [8, с. 990].

Также имеются другие факторы, кроме края резекции, которые влияют на частоту рецидивов. В метаанализе, включившем 7174 пациента, получавших органосохранную хирургию и ЛТ, относительный риск (OP) развития местного рецидива у пациентов с опухолями, экспрессирующими гормональные рецепторы или HER2, составил 0,49 (95\%ный доверительный интервал [ДИ] 0,33-0,73) по сравнению с пациентами с тройным негативным раком $(\mathrm{OP}=0,33)$ [9, с. 840]. Влияние биологии опухоли на местный рецидив настолько глубокое, что статистически значимые различия в частоте наблюдаются среди опухолей размером 1 см и менее в зависимости от рецептора эстрогена (РЭ), рецептора прогестерона (РП) и статуса HER2 [10, с. 715]. Даже в подгруппе пациентов с отрицательными лимфоузлами, но с положительными РП и отрицательным HER2 показаны химиотерапия и тамоксифен - Маmounas и соавт. [11, с. 1682].

Другим фактором, существенно влияющим на частоту локального рецидива, является широкое применение адъювантной системной терапии. В одном метаанализе было показано, что тамоксифен в течение 5 лет уменьшил ОР рецидива до 0,47 по сравнению с плацебо $(\mathrm{OP}=0,63)$ [12, с. 2091]. Достижения таргетной терапии также улучшили результаты выживаемости. Например, применение ингибиторов ароматазы или продолжительной гормональной терапии снизило ОР локального рецидива с 0,83 до 0,50 по сравнению с только тамоксифеном [13, с. 22]. Добавление трастузумаба к химиотерапии снижает ОР локального рецидива до 0,47 по сравнению с одной только химиотерапией, что приводит к снижению 3 -летней частоты локального рецидива с $7 \%$ до $1 \%$ (p=0,01) [14, с. 1988]. В 
крупном анализе (n=86 598) пациентов, участвовавших в 53 рандомизированных исследованиях за период с 1990 по 2011 гг., доля локального рецидива в общем числе уменьшилась с $30 \%$ до $15 \%(\mathrm{p}<0,001)(15)$.

Учитывая значительные различия в частоте реэксцизий, а также изменения в понимании биологии РМЖ, Общество Хирургической Онкологии (SSO) и Американское Общество Радиационной онкологии (ASTRO) созвали совещание многодисциплинарной группы в 2013 г., чтобы решить вопрос, каков должен быть размер ширины края резекции, который минимизирует риск локального рецидива у больных с инвазивным РМЖ при сохранной операции и ЛТ. Как часть доказательной базы для этого консенсуса метаанализ включал 33 исследования с охватом 28162 пациентов и 1506 случаев локального рецидива с медианой наблюдения 79,2 месяцев. Частота локального рецидива составила 5,3\%. Основные выводы метаанализа заключались в том, что положительные или неизвестные размеры краев резекции повышали риск локального рецидива (отношение шансов $=2,44$ ) по сравнению с отрицательным краем резекции и что не наблюдалось никакой корреляции между увеличением отрицательной ширины края резекции (1 мм, 2 мм, 5 мм) и снижением риска локального рецидива [16, с. 730]. Главный вопрос, затронутый в консенсусе, заключался в том, можно ли определить «оптимальную» ширину отрицательного края резекции. Метаанализ, изучавший ширину полей 1, 2 и 5 мм, включал 19 исследований и 13081 пациента с 753 случаями локального рецидива при медиане наблюдения 8,7 лет. Статистическая связь между увеличением ширины края резекции и снижением частоты локального рецидива не наблюдалась $(\mathrm{p}=0,90)$. Поправки на возрастные вариации, эндокринную терапию, лучевую стимуляцию, использование повторного иссечения и срок первого рецидива по сравнению с любым другим сроком не изменили этих результатов. Группа пришла к выводу о том, что отрицательные поля, определяемые как отсутствие чернил на опухоли, минимизируют риск развития локального рецидива, однако более широкие четкие поля не снижают значительно этот риск [17, с. 553].

В консенсусном заявлении рассматривались конкретные клинические обстоятельства, представляющие интерес для хирургов, включая влияние гистологических особенностей опухоли, таких как инфильтрирующая дольковая карцинома или дольковая карцинома in situ или наличие обширного внутрипротокового компонента. Риск развития локального рецидива не отличается у пациентов с инвазивным дольковым и инвазивным протоковым раком [18, с. 455]. В исследовании 382 пациентов с дольковой карциномой при сравнении полей $<1$ см и $\geq 1$ см достоверных различий в локальном рецидиве отмечено не было [19, с. 580]. Кроме того, большинство классических инфильтрирующих дольковых карцином являются РЭположительными, эндокринно-чувствительными опухолями, и эти пациенты испытают 
преимущества эндокринной терапии. Исследования, изучающие влияние присутствия дольковой карциномы на границах резекции, не показали связи между опухолью на границе и локальным рецидивом [20, с. 2263]. Таким образом, консенсус-группа по изучению краев резекции пришла к выводу, что при широких отрицательных полях, отсутствии чернил на опухоли и классическом варианте дольковой карциномы повторное иссечение не показано.

В отличие от долькового типа, при протоковом варианте наличие опухоли в крае резекции связано с повышенным риском локального рецидива. Раковые опухоли с экстенсивным интрадуктальным компонентом имели повышенный риск развития локального рецидива по сравнению с опухолями без него, и значительное число пациентов с экстенсивным интрадуктальным компонентом имели дополнительные очаги на расстоянии $\geq$ 2 см от расположения опухоли [21, с. 1746].

Биологические особенности, такие как возраст пациента и подтип опухоли, могут повлиять на рекомендации по значению края резекции. Молодой возраст, обычно < 40 лет, ассоциировался с повышенным риском развития локального рецидива в более ранних исследованиях [22, с. 1716]. В последнее время частота локального рецидива у более молодых женщин снизилась до уровней, сопоставимых с аналогичными показателями у их старших коллег, в основном из-за более широкого применения химиотерапии и таргетной терапии [23, с. 3093]. Кроме того, большие поля, полученные при мастэктомии, не уменьшают частоту локального рецидива или выживаемость у молодых женщин [24, с. 517].

Пациенты с тройным негативным РМЖ имеют более высокий риск развития локального рецидива, чем пациенты с другими подтипами [9, с. 840]. Исследования, сравнивающие показатели локального рецидива после сохранной операции + ЛТ и мастэктомии у пациентов с тройным негативным раком, не подтверждают этого предположения [25, с. 2852, 26, с. 3476]. Pilewskie и соавт. исследовали уровень локального рецидива у 535 пациентов с тройным негативным раком (сохранная операция + ЛТ) и обнаружили, что 5-летний риск локального рецидива при ширине края резекции $\leq 2$ мм составил 4,7\% против $3,7 \%$ у пациентов с более широкими размерами $(\mathrm{p}=0,11)$ [27, с. 1214]. Это и предыдущее обсуждение относительно молодых женщин подчеркивает факт того, что расширенная хирургия не влияет на неблагоприятную биологию опухоли.

Наличие рака в непосредственной близости к краю резекции связано с риском остаточной опухоли. В этом случае должны иметь место тщательный анализ и оценка остаточной опухоли (массы опухоли, близость опухоли к краю и картина ее роста).

Руководство по инвазивному РМЖ было опубликовано в Интернете в ноябре 2013 г. и одновременно опубликовано в Annals of Surgical Oncology, Journal of Clinical Oncology и International Journal of Radiation Oncology Biology and Physics в мае 2014 г. Опрос членов 
американского общества хирургов молочной железы в октябре 2014 г. показал, что только 1,3\% из 777 респондентов регулярно повторно иссекают при ширине $<2$ мм. Чаще хирурги производили повторное иссечение в сложных клинических сценариях, таких как множественные близкие границы или наличие экстенсивного интрадуктального компонента [28, с. 3256]. В целом количество повторных иссечений в США снизилось с $19 \%$ до 13\%, что позволило сэкономить 30 млн долл. США в год на системе здравоохранения, использующей программу Medicare [29, с. 3239].

Сокращение опухоли при РМЖ в ответ на неоаъювантную терапию происходит двумя путями: концентрическим и в виде ячеек или картечи. У пациентов с гистологически полным ответом или концентрическим сокращением опухоли учет ширины края не отличается от такового при первичной хирургической обработке. У пациентов с сотовым рисунком определение соответствующей степени резекции более проблематично. Исследователи из онкологического центра Университета штата Техас MD Anderson показали, что при многомерном анализе сотовой структуры ответа остаточная болезнь $>2$ см и клиническая болезнь N2 или N3 были статистически значимыми предикторами локального рецидива после органосохранного лечения РМЖ [30, с. 2303].

Bce предостережения, касающиеся отсутствия стандартного подхода к патологической оценке полей при инвазивном раке, применимы к экстенсивным внутрипротоковым карциномам, а данные рандомизированных исследований уровня I о влиянии увеличения ширины полей на локальный рецидив отсутствуют. Существует несколько причин, по которым оптимальная ширина полей для этого типа может отличаться от инвазивного рака. Было продемонстрировано, что экстенсивная внутрипротоковая карцинома развивается в протоках прерывисто, что способствует возникновению остаточной опухоли. Поэтому ретроспективные исследования показали, что отрицательный край резекции $\geq 1$ см и ЛТ снижают частоту локального рецидива. Это служило основой решения SSO, ASTRO и ASCO о разработке междисциплинарного, основанного на фактических данных консенсусного руководства для этих типов рака в 2015 г. Это руководство опирается на большой метаанализ, который включал 7883 пациента в 20 исследованиях и 865 случаев локального рецидива. Медиана наблюдения составила 78,3 месяца, а частота локального рецидива - 8,3\% [31, с. 3811]. Из-за неоднородности исследований был проведен как частотный, так и байесовский сетевой анализ, который подтвердил, что отрицательные поля по сравнению с положительными полями уменьшили риск локального рецидива примерно на $50 \%$.

Заключение. Таким образом, рак молочной железы остается актуальной проблемой в онкологии. Несмотря на имеющиеся достижения, результаты еще остаются не вполне 
удовлетворительными. На результаты лечения влияют многие факторы, такие как биологические особенности опухоли, стадия опухолевого процесса, гормональный статус, возраст, гистологическая структура опухоли, выбор метода лечения и др. В последнее время мастэктомию упорно вытесняют органосохранные операции. Такой консервативный подход с использованием лучевой и гормонотерапии с учетом гормонального статуса позволил достичь хороших результатов в лечении. Однако до сих пор нет единого мнения, каковы должны быть величины ширины размеров края резекции при ранних стадиях рака молочной железы. Обзор литературных данных показал, что, к сожалению, количество повторных иссечений еще наблюдается, несмотря на четкую маркировку операционного поля. Диапазон дискутируемой разными авторами ширины резекции широк и колеблется от 1 мм до 10 мм. Однако метаанализы многочисленных исследований показали, что отрицательным край должен считаться при ширине 2 мм и менее.

\section{Список литературы}

1. Anderson S.J., Wapnir I.L. Prognosis after ipsilateral breast tumor recurrence and locoregional recurrences in patients treated by breast-conserving therapy in five National Surgical Adjuvant Breast and Bowel Project protocols of node-negative breast cancer. J. Clin. Oncol. 2009. V.27 (15). P.2466-2473.

2. Wapnir I.L., Anderson S.J., Mamounas E.P., Geyer C.E. Jr., Jeong J.H., Tan-Chiu E., Fisher B., Wolmark N. Prognosis after ipsilateral breast tumor recurrence and locoregional recurrences in five National Surgical Adjuvant Breast and Bowel Project node-positive adjuvant breast cancer trials. J.Clin. Oncol. 2006. V.4 (13). P.2028-2037.

3. Van Zee K.J., Subhedar P. Relationship Between Margin Width and Recurrence of Ductal Carcinoma In Situ: Analysis of 2996 Women Treated With Breast-conserving Surgery for 30 Years. Ann Surg. 2015. P. 262(4). P.623-631.

4. Azu M., Abrahamse P., Katz S.J., Jagsi R., Morrow M. What is an adequate margin for breast-conserving surgery? Surgeon attitudes and correlates. Ann. Surg. Oncol. 2010. V.17(2). P.558-63.

5. Morrow M., Jagsi R., Alderman A.R., Griggs J.J., Hawley S.T., Hamilton A.S., Graff J.J., and Katz S.J. Surgeon recommendations and receipt of mastectomy for treatment of breast cancer. JAMA. 2009. V. 302(14). P.1551-1556.

6. McCahill L.E., Single R.M., Aiello Bowles E.J., Feigelson H.S., James T.A., Barney T., Engel J.M., Onitilo A.A. Variability in reexcision following breast conservation surgery. JAMA. 2012. V.307 (5). P.467-475. 
7. Chagpar A.B., Killelea B.K., Tsangaris T.N., Butler M., Stavris K., Li F., Yao X., Bossuyt V., Harigopal M., Lannin D.R., Pusztai L., Horowitz N.R. A Randomized, Controlled Trial of Cavity Shave Margins in Breast Cancer. N. Engl. J. Med. 2015. V. 373(6). P.503-510.

8. Holland R., Veling S.H. Histologic multifocality of Tis, T1-2 breast carcinomas. Implications for clinical trials of breast-conserving surgery. Cancer. 1985. V.56(5). P.979-990.

9. Lowery A.J., Kell M.R., Glynn R.W., Kerin M.J., Sweeney K.J. Locoregional recurrence after breast cancer surgery: a systematic review by receptor phenotype. Breast Cancer Res Treat. 2012. V.133 (3). P.831-841.

10. Cancello G., Maisonneuve P., Rotmensz N., Viale G., Mastropasqua M.G., Pruneri G., Montagna E., Dellapasqua S., Iorfida M., Cardillo A., Veronesi P., Luini A., Intra M., Gentilini O., Scarano E., Goldhirsch A., Colleoni M. Prognosis in women with small (T1mic,T1a,T1b) nodenegative operable breast cancer by immunohistochemically selected subtypes. Breast Cancer Res Treat. 2011. V. 127(3). P.713-720.

11. Mamounas E.P., Tang G., Fisher B., Paik S., Shak S., Costantino J.P., Watson D., Geyer C.E. Jr., Wickerham D.L., Wolmark N. Association between the 21-gene recurrence score assay and risk of locoregional recurrence in node-negative, estrogen receptor-positive breast cancer: results from NSABP B-14 and NSABP B-20. J. Clin. Oncol. 2010. V. 28(10). P.1677-1683.

12. Clarke M., Collins R., Darby S., Davies C., Elphinstone P., Evans V., Godwin J., Gray R., Hicks C., James S., MacKinnon E., McGale P., McHugh T., Peto R., Taylor C., Wang Y.; Early Breast Cancer Trialists' Collaborative Group (EBCTCG).Early Breast Cancer Trialists' Collaborative Group. Effects of radiotherapy and of differences in the extent of surgery for early breast cancer on local recurrence and 15-year survival: an overview of the randomised trials. Lancet. 2005. V. 366(9503). P.2087-2106.

13. Mannino M., Yarnoldv J. Accelerated partial breast irradiation trials: diversity in rationale and design. Radiother Oncol. 2009. V. 91(1). P.16-22.

14. Kiess A.P., McArthur H.L., Mahoney K., Patil S., Morris P.G., Ho A., Hudis C.A., McCormick B. Adjuvant trastuzumab reduces locoregional recurrence in women who receive breast-conservation therapy for lymph node-negative, human epidermal growth factor receptor 2positive breast cancer. Cancer. 2012. V. 118(8). P.1982-1988.

15. Bouganim N., Tsvetkova E., Clemons M., Amir E. Evolution of sites of recurrence after early breast cancer over the last 20 years: implications for patient care and future research. Breast Cancer Res Treat. 2013. V. 139(2). P.603-606.

16. Houssami N., P. Macaskill, Marinovich M.L., Morrow M. The association of surgical margins and local recurrence in women with early-stage invasive breast cancer treated with breastconserving therapy: a meta-analysis. Ann. Surg. Oncol. 2014. V. 21(3). P.717-730. 
17. Moran M.S., Schnitt S.J., Giuliano A.E., Harris J.R., Khan S.A., Horton J., Klimberg S., Chavez-MacGregor M., Freedman G., Houssami N., Johnson P.L., Morrow M. Society of Surgical Oncology-American Society for Radiation Oncology consensus guideline on margins for breastconserving surgery with whole-breast irradiation in stages I and II invasive breast cancer. Int. J. Radiat. Oncol. Biol. Phys. 2014. V. 88(3). P.553-564.

18. Biglia N., Maggiorotto F., Liberale V., Bounous V.E., Sgro L.G., Pecchio S., D'Alonzo M., Ponzone R. Clinical-pathologic features, long term-outcome and surgical treatment in a large series of patients with invasive lobular carcinoma (ILC) and invasive ductal carcinoma (IDC) Eur. J. Surg. Oncol. 2013. V. 39(5). P.455-460.

19. Galimberti V., Maisonneuve P., Rotmensz N., Viale G., Sangalli C., Sargenti M., Brenelli F., Gentilini O., Intra M., Bassi F., Luini A., Zurrida S., Veronesi P., Colleoni M., Veronesi U. Influence of margin status on outcomes in lobular carcinoma: experience of the European Institute of Oncology. Ann Surg. 2011. V. 253(3). P. 580-584.

20. Ciocca R.M., Li T., Freedman G.M., Morrow M. Presence of lobular carcinoma in situ does not increase local recurrence in patients treated with breast-conserving therapy. Ann Surg Oncol. 2008. V. 15(8). P.2263-2271.

21. Schnitt S.J., Abner A., Gelman R., Connolly J.L., Recht A., Duda R.B., Eberlein T.J., Mayzel K., Silver B., Harris JR. The relationship between microscopic margins of resection and the risk of local recurrence in patients with breast cancer treated with breast-conserving surgery and radiation therapy. Cancer. 1994. V. 74(6). P.1746-1751.

22. Darby S., McGale P., Correa C., Taylor C., Arriagada R., Clarke M., Cutter D., Davies C., Ewertz M., Godwin J., Gray R., Pierce L., Whelan T., Wang Y., Peto R. Early Breast Cancer Trialists' Collaborative G. Effect of radiotherapy after breast-conserving surgery on 10-year recurrence and 15-year breast cancer death: meta-analysis of individual patient data for 10,801 women in 17 randomised trials. Lancet. 2011. V. 378(9804). P.1707-1716.

23. van Laar C., van der Sangen M.J., Poortmans P.M., Nieuwenhuijzen G.A., Roukema J.A., Roumen R.M., Tjan-Heijnen V.C., Voogd A.C. Local recurrence following breast-conserving treatment in women aged 40 years or younger: trends in risk and the impact on prognosis in a population-based cohort of 1143 patients. Eur. J. Cancer. 2013. V. 49(15). P.3093-3101.

24. Cao J.Q., Truong P.T., Olivotto I.A., Olson R., Coulombe G., Keyes M., Weir L., Gelmon K., Bernstein V., Woods R., Speers C., Tyldesley S. Should women younger than 40 years of age with invasive breast cancer have a mastectomy? 15-year outcomes in a population-based cohort. Int. J. Radiat. Oncol. Biol. Phys. 2014. V. 90(3). P.509-517.

25. Abdulkarim B.S., Cuartero J., Hanson J., Deschênes J., Lesniak D., Sabri S. Increased risk of locoregional recurrence for women with T1-2N0 triple-negative breast cancer treated with 
modified radical mastectomy without adjuvant radiation therapy compared with breast-conserving therapy. J. Clin. Oncol. 2011. V.29(21). P.2852-2858.

26. Zumsteg Z.S., Morrow M., Arnold B., Zheng J., Zhang Z., Robson M., Traina T., McCormick B., Powell S., Ho A.Y. Breast-conserving therapy achieves locoregional outcomes comparable to mastectomy in women with T1-2N0 triple-negative breast cancer. Ann. Surg. Oncol. 2013. V. 20(11). P.3469-3476.

27. Pilewskie M., Ho A., Orell E., Stempel M., Chen Y., Eaton A., Patil S., Morrow M. Effect of margin width on local recurrence in triple-negative breast cancer patients treated with breastconserving therapy. Ann. Surg. Oncol. 2014. V. 21(4). P.1209-1214.

28. DeSnyder S.M., Hunt K.K., Smith B.D., Moran M.S., Klimberg S., Lucci A. Assessment of Practice Patterns Following Publication of the SSO-ASTRO Consensus Guideline on Margins for Breast-Conserving Therapy in Stage I and II Invasive Breast Cancer. Ann Surg Oncol. 2015. V. 22(10). P.3250-3256.

29. Greenup R.A., Peppercorn J., Worni M., Hwang E.S. Cost implications of the SSO-ASTRO consensus guideline on margins for breast-conserving surgery with whole breast irradiation in stage I and II invasive breast cancer. Ann. Surg. Oncol. 2014. V. 21(5). P.1512-1514.

30. Chen A.M., Meric-Bernstam F., Hunt K.K. Breast conservation after neoadjuvant chemotherapy: the MD Anderson cancer center experience. J. Clin. Oncol. 2004. V. 22(12). P.23032312.

31. Marinovich M.L., Azizi L., Macaskill P., Irwig L., Morrow M., Solin L.J., Houssami N. The Association of Surgical Margins and Local Recurrence in Women with Ductal Carcinoma In Situ Treated with Breast-Conserving Therapy: A Meta-Analysis. Ann. Surg. Oncol. 2016. V.23(12). P.3811-3821. 